\title{
A study on clinical presentation and outcome of scorpion sting in children
}

\author{
S. Pazhanisamy ${ }^{1, *}$, J. Madhavan ${ }^{2}$ \\ Assistant Professor, Dept. of Paediatrics, Thanjavur Medical College, Dr. M. G. R. University, Chennai, Tamil Nadu, India
}

*Corresponding Author:

Email: drrspmd@gmail.com

\begin{abstract}
Introduction and objectives: Scorpion sting is a frequent, life threatening medical emergency in children. They constitute a significant public health problem in many underdeveloped countries, including India. This study was done to analyse the clinical presentation and outcome of scorpion sting in children

Material and Methods: This is an observational study of 141 cases of scorpion sting, admitted at Government Raja Mirasdar Hospital, Thanjavur. The clinical presentation and outcome were studied.

Results: Scorpion sting is a common, pediatric emergency in our area. Rural male children, from lower socioeconomic groups, aged between 1-3 years (35\%) and 7-12 years (34\%) were most commonly affected. Pain at the site of sting (89\%) and cold peripheries $(77 \%)$ were the most common presenting symptom and sign respectively. Myocarditis (9\%) and pulmonary edema (4\%), were also frequently encountered. Complication were more common in younger children and in cases who received the first dose of prazosin late i.e. (After 6 hours). One case succumbed to the death $(0.7 \%)$, but majority of the cases $(99 \%)$, recovered without sequelae.

Conclusion: Scorpion sting is a serious, potentially fatal emergency in our area. Peripheral circulatory failure is common. Cardiovascular manifestations are life threatening. Scorpion stings constitute a "occupational hazard" for children employed as agricultural labourers. Administration of prazosin, as early as possible, is probably, the single most effective intervention in preventing complication and mortality.
\end{abstract}

Keywords: Scorpion stings, Pulmonary edema, Clinical features, Envenomation, Prazosin.

\section{Introduction}

Scorpion sting is a frequent, life threatening, time limiting medical emergency in children. They constitute a significant public health problem in many under developed countries including India. Scorpion sting is more common in rural parts of India. They are nocturnal in habit. There are about 1500 species of scorpion worldwide, among them 50 species are dangerous to human beings. Almost all lethal scorpions belong to Buthidae family. Clinical manifestations of scorpion sting include vomiting, profuse sweating, loss of sphincter control, diarrhea and priapism. Hypertension, tachycardia, myocardial dysfunction and pulmonary edema are common complication. The clinical manifestations of scorpion sting envenomation are due to massive release of sympathetic and para sympathetic neuro transmitters. Numerous envenomation goes unreported and the true incidence is not known. ${ }^{1}$ Case fatality rates vary widely among different regions from $3-22 \%$ and over the years with improvement in management protocols, there has been a dramatic reduction in mortality. This study deals with various clinical presentations and outcome of scorpion sting in children.

\section{Material and Methods Patient population}

This was a descriptive observational hospital-based study conducted in the department of pediatrics, Government Raja Mirasdhar Hospital, Thanjavur Medical College, Thanjavur. During the period of
January 2014 to July 2014, a total of 141 cases of scorpion sting admitted with history of unknown bite coupled with classic clinical manifestations of scorpion sting were included in the study. Children with scorpion sting treated elsewhere and referred to higher center whose initial presentation and treatment details were not available and children with co-morbid illness like congenital heart disease and severe respiratory illness were excluded

\section{Methods}

History and clinical presentation at the time of admission were noted using the proforma. Follow up of the clinical parameters such as duration to achieve normalcy of heart rate, respiratory rate, Blood pressure, priapism and overall wellbeing of the child were recorded till discharge or death.

All the patients with peripheral circulatory failure were treated with tablet prazosin 30 microgram per kilogram per dose, intravenous fluid and supportive measures. Second dose of prazosin was given after three hours and third dose after six hours till peripheries became warm. Myocarditis was treated with oxygen, intravenous fluids, prazosin, dobutamine infusion. Pulmonary edema was treated with oxygen, prazosin, dobutamine, frusemide and mechanical ventilation if indicated. All cases were managed and monitored for the complications and treated accordingly. 


\section{Results}

There were 141 children admitted with scorpion sting during this study period. Among 141 children,50 (35\%) were in the age group of 1 year to 3 years, 47 (34\%) children were in the age group of 7 years to 12 years, 36(25\%) were in the age group of 4 years to 6 years and only $8(6 \%)$ were below 1 year. There were 87 (62\%) males and 54 (38\%) females with male to female ratio of 1.5:1. Among the cases we studied, 105(74\%) scorpion sting cases were from rural areas, remaining 36 (26\%) cases were admitted from urban area. Scorpion sting cases were noted in all classes of Socio Economic Status, except class 5. Class 1(38\%) and class $2(33 \%)$ together accounted $71 \%$ of the cases. The majority of stings occurred during night time (81 cases (57\%)).The most of the stings were sustained on the extremities (91\%).Only 9\%of stings were sustained were on other parts of the body like head, face abdomen, etc.

\section{Clinical analysis in patient with scorpion sting}

During the study period only $3.5 \%$ of the cases were admitted with altered sensorium, remaining $96.5 \%$ cases did not have altered sensorium. About $89 \%$ of cases had pain over the stings site, $62 \%$ of cases had sweating and $29 \%$ of cases had salivation. $18 \%$ of cases had vomiting and $9 \%$ of cases had swelling over the sting site. Priapism is found only in 8 cases (9\%). Most of the cases had normal heart rate $(53 \%)$, respiratory rate $(97 \%)$ and blood pressure (75.5\%).Most of scorpion sting cases had cold peripheries, only $8 \%$ cases had breathing difficulty. The most severe complication of scorpion sting is myocarditis and pulmonary edema which occurred in $9 \%$ and $4 \%$ of the cases respectively in our study.

In our study, $57 \%$ of the cases received the first dose of prazosin from 1 to 6 hours. For the reversal of autonomic storm, $37 \%$ of cases required single dose of prazosin, $39 \%$ of cases required two or three doses of prazosin. The autonomic storm was reversed within 12 hours in $65 \%$ of the cases, for the remaining $35 \%$ of the cases it took more than 12 hours for the reversal of autonomic storm. During the study period only $8 \%$ of cases needed inotropic support. Duration of stay in the hospital is less than 5 days in $93 \%$ cases. The average stay duration is 2 to 5 days.

\section{Discussion}

Scorpion sting is an acute life threatening, time limiting medical emergency of villages. We studied 141 cases of scorpion sting, admitted in our hospital from January 2014 to July 2014 and our observations are discussed below.

\section{Age and Sex distribution}

Children between 6-12 years of age are more exploratory and tend to wander outside their homes in the darkness and hence are more susceptible. Mahadevan $\mathrm{S}$ in 1981, reported a series of 100 cases of scorpion sting in children and reported a similar age distribution ${ }^{2}$. There was a male preponderance in cases studied by us. This has also been noted in the past by various authors ${ }^{3}$. This could be due to higher inquisitive nature of boys and they wander more outside than the girls.

\section{Urban and Rural area}

Majority of the cases (105 Cases=75\%) were from rural areas. Like snake bite, Scorpion sting is mainly a rural emergency ${ }^{1}$. Children from the rural areas are at highest risk for accidental contact with scorpions.

\section{Housing and Socioeconomic status}

The incidence of scorpion sting is higher in children living in kutcha houses. Kutcha houses have mud floors, walls and thatched roofs. Scorpion inhabit the cervices and underground burrows in dwellings and these houses provide a safe heaven for them. In contrast, pukka houses with tiled floors and cemented walls and roofs are safe, which was similar to Rajeshwari et al, Sivaprakasam et al's observation. ${ }^{8}$ A higher incidence of sting was noted in lower socioeconomic status. The high incidence in this group is due to the type of housing and farms nearby their houses.

\section{Site and Timing of sting}

Although any part of the body can be exposed to sting, in $91 \%$ of the cases in our study, the sting was sustained on the extremities. This was comparable to Mahaba et al's observation. Night time stings were more common with a number of eighty one cases. Our study findings correlate with the study done by Clark et al, Mahadevan et al, where the incidence of scorpion sting were more common during night time. ${ }^{4}$

\section{Incidence of Signs and Symptoms in scorpion sting}

Pain at the sting site was the commonest complaint and was invariably present in most of the cases. The high incidence of pain was also noted in previous studies. ${ }^{8}$ Profuse sweating was noted in $62 \%$ of the cases. Vomiting and excessive salivation in $18 \%$ and $29 \%$ of cases respectively. Local swelling was noted only in $9 \%$ of the cases. Cold peripheries were noted in $77 \%$ of cases. Eight patient had priapism. Bawaskar et al noted priapism in 10 of the cases and observed it to be one of the important cardiac premonitory signs. ${ }^{7}$

\section{Scorpion sting presented with "autonomic storm"}

Scorpion venom delays the closing of neuronal sodium channels, and the inhibition of potassium channels both leading to intense persistent stimulation of autonomic nerves predominantly alpha receptors leading to massive release of neurotransmitters from adrenal medulla stimulating parasympathetic and sympathetic nerve endings thus initiating autonomic storm. In scorpion sting, $60 \%$ to $70 \%$ of the cases presented with "autonomic storm" characterized by cold extremities, tachycardia, hypotension or hypertension.in our study hypertension was noted in $21 \%$ of the cases. Incidence 
of hypertension in scorpion Indian studies varies from $12.6 \%$ to $29 \%$ and usually seen within $4-8$ hours after the sting. ${ }^{8}$ Hypotension was noted in our study only 6 cases, hypotension can occur within 1-2 hours after the sting, due to loss of fluid and also within 4-8 hours due to left ventricular dysfunction. ${ }^{1}$ Tachycardia was present in $43.5 \%$ of cases at the time of admission and bradycardia was noted only in $4 \%$ of the cases at the time of admission. Bradycardia is an early finding in autonomic storm due to cholinergic over activity and has been reported in $8-15 \%$ of cases. ${ }^{2,8,9}$

\section{Incidence of myocarditis and pulmonary edema}

In our study five patients presented with altered sensorium, among them three patients developed myocarditis, two patients developed pulmonary edema and one patient among pulmonary edema was expired. Out of 141 patients twelve children had scorpion sting with myocarditis, among them 10 patients $(83 \%)$ were less than four years of age. Six patients had pulmonary edema, among them five patients were less than six years of age. The myocarditis and pulmonary edema more common in young children. Similar findings were observed by Bawaskar. H. S et al and Biswal, N.et al. ${ }^{13}$ Both cardiogenic and non cardiogenic factors have been implicated in pulmonary edema secondary to scorpion sting The dose of the venom relative to the weight is obviously higher in younger children, thus rendering them susceptible to more severe envenomation and greater complication.

\section{Time for Reversal of Autonomic storm}

Prazosin, an alpha adrenoceptor antagonist is a physiological and pharmacological antidote of scorpion venom. Cardiovascular morbidity depends on the time interval between sting and administration of prazosin. A consensus regarding early use of prazosin has been established. Complication were noted less frequently in children who received a dose prazosin early $(<6$ hours of sting). This finding is comparable to studies done elsewhere in India, which show that early and effective administration of prazosin significantly reduced the incidence of complications and mortality. ${ }^{11,12}$

\section{Mortality in Scorpion sting}

Death due to scorpion sting occurs mainly due to massive pulmonary edema and Congestive Cardiac Failure with cardiogenic shock. The mortality due to scorpion sting has dramatically declined over the years from $68 \%$ to less than $1 \% .^{1,2,8}$ Similar findings were observed in our study also, out of 141 cases only one case expired $(0.07 \%)$. Early administration of prazosin and improved management practices were the important factors responsible for decline.

\section{Conclusion}

Scorpion sting is a most common life threatening emergency among children in our area, maximum number of cases were in the age group of 1-3 years and 7-12 years. Majority of cases were from rural areas, sustained in kutcha house and more number of cases noted among low socioeconomic status. Stings sustained indoor were more than outdoors and mostly during the night time. Pain at the sting site was noted in majority of cases. Peripheral circulatory failure was found in $77 \%$ of the cases. Myocarditis was found in $9 \%$ of the cases and pulmonary edema was found in $4 \%$ of the cases. Most of the cases recovered without any sequelae. During the study period one death occurred due to myocarditis and pulmonary edema. Case fatality rate was less than one percent. Early and effective prazosin therapy, good supportive care, close monitoring and management of complications can limit the resulting morbidity and mortality significantly.

\section{Statistical Analysis}

Statistical analysis was done by descriptive statistics using simple ratio and percentages. Microsoft office 2010 was used to generate the tables.

\section{Ethical consideration and approval}

Preceding the study, approval for the study was obtained from the institutional research ethical committee of Thanjavur medical college. Permission to perform this study was obtained from the Raja Mirasdhar Hospital, Thanjavur medical college authority.

Funding: No funding sources.

Conflict of interest: None declared.

Ethical approval: The study was approved by the institutional ethics committee.

\section{References}

1. Mahadevan S. Scorpion Sting. Indian Pediatr 2000;37:504-14.

2. Mahadevan S, Choudhary P, Puri RK, Srinivasan Scorpion envenomation and the role of lytic cocktail in its management. Indian J Pediatr 1981;48:757-61.

3. Cheng D, Dattaro JA, Yakobi R. Scorpion sting. Cited on 2005 June 23; 24 screens: Available from: URL://www.emedicine.com/med/topic2081.htm.Accesse d August 28, 2007.

4. Clark RF. Scorpion Envenomation. In: Clinical Toxicology. Ford MD, Delaney KA, Ling LJ, Erickson J, edt., $1^{\text {st }}$ edn., W.B. Saunders company. Pennsylvania 2004:290-93.

5. Mahaba HMA. Scorpion Sting Syndrome: Epidemiology, clinical presentation and management of 2240 cases. Eastern Mediterranean Health J 1997;3(1):82-9.

6. Dittrich K, Power AP, Smith NA, Scorpion sting syndrome-A Ten year Experience. Ann Saudi Med 1995;15(2):148-55.

7. Bawaskar HS. Diagnostic cardiac premonitory signs and symptoms of red scorpion sting. Lancet 1982;1(8271):552-4.

8. Rajarajeshwari G, Sivaprakasam S, Viswanathan J. Morbidity and Mortality pattern in Scorpion Stings. $J$ Indian Med Assoc 1979;73(7\&8):123-26. 
9. Singh DS, Bisht DB, Sukumar G, Muralidhar K. Scorpion stings in adult South Indians at Pondicherry Indian M A 1979;72(10):234-37.

10. Santhanakrishnan BR. Scorpion stings. Indian Pediatr 2000;37:1154-57.

11. Bawaskar HS, Bawaskar PH. Clinical profile of severe scorpion envenomation in children at rural setting. Indian Peadiatr 2003;40:1072-76.

12. Bawaskar HS, Bawaskar PH. Prazosin in the management of cardiovascular manifestations of scorpion sting. Lancet 1986;1(8479):510-11.
13. Biswal N, Bashir Rani A, Murmu Uday C, Mathai B, Balachander J, Srinivasan S. Outcome of scorpion sting envenomation after a protocol guided therapy. Indian $J$ Pediatr 2006;73:577-82.

How to cite the article: Pazhanisamy S., Madhavan J. A study on clinical presentation and outcome of scorpion sting in children. IP Int J Med Paediatr Oncol 2018;4(3):110113. 